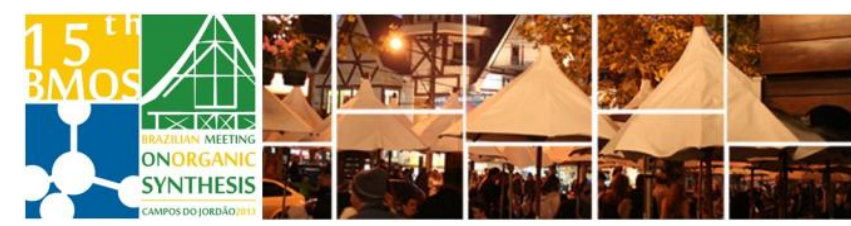

\title{
Preparation of New Prenylated (E)-Chalcones
}

\author{
*Mariana Bastos dos Santos ${ }^{a}$, Fernanda Patricia Gullob, Luiz Antonio Dutrab, Maria \\ José Soares Mendes-Giannini ${ }^{b}$, Ana Marisa Fusco-Almeida ${ }^{b}$, Luis Octávio Regasini ${ }^{a}$ \\ ${ }^{a}$ Departamento de Química e Ciências Ambientais - Instituto de Biociências, Letras e Ciências Exatas - São \\ José do Rio Preto - UNESP; ${ }^{b}$ Departamento de Análises Clínicas - Faculdade de Ciências Farmacêuticas - \\ Araraquara - UNESP \\ *mariana19bsantos@gmail.com
}

Keywords: prenylated chalcones, prenylation, Claisen rearrangements.

\section{INTRODUCTION}

Chalcones comprise one of the main classes of natural products, and their structure is an interesting scaffold for different chemical modifications, including the functional group interconversions. ${ }^{1,2}$ Among the different groups that are correlated to bioactivity of chalcones, the side prenyl chains seem to be related to plasmatic membrane anchorage. For this reason, we have synthesized a series of 20 new O-prenylated chalcones, including isoprenylated, geranylated and farnesylated derivatives. In addition, these compounds were evaluated as antifungal agents.

\section{RESULTS AND DISCUSSION}

The $(E)$-chalconic building block preparation was carried out by Claisen-Schmidt aldol reaction, under basic or acid catalysis, generating monohydroxylated chalcones (Figure 1).

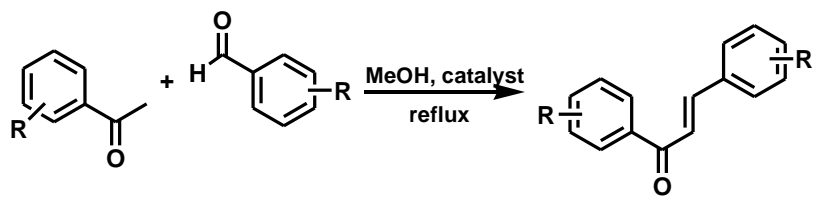

Figure 1. Preparation of (E)-chalconic building blocks (catalyst $=\mathrm{H}_{2} \mathrm{SO}_{4}$ or $\mathrm{NaOH} ; \mathrm{R}=\mathrm{H}$ or hydroxyl)

For preparation of the prenylated $(E)$-chalcone derivatives was used alkylation reactions with terpenyl bromides in alkaline medium, and reflux (Figure 2).<smiles></smiles>

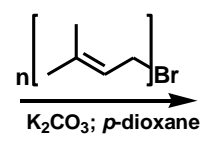<smiles>O=C(/C=C/c1ccccc1)c1ccc(Br)cc1</smiles>

Figure 2. Preparation of prenylated $(E)$-chalcones $[\mathrm{R}=\mathrm{H}$ or hydroxyl; $R_{1}=H$, isoprenyl $(n=1)$, geranyl $(n=2)$ or farnesyl $(n=3)$ ]
In general, the preparation of the monohydroxylated chalcones demonstrated yields between $56 \%$ and $86 \%$, and the prenylated chalcones were obtained in satisfactory yields (57\%-92\%). Despite the formation of the designed $O$-prenylated chalcones, some $C$ prenylated derivatives as byproducts from Claisen rearrangements were detected, requiring additional purification steps, including Column Chromatography and Preparative Thin Layer Chromatography.<smiles>C=CC(C)(C)c1ccccc1O</smiles>

Figure 3. Conversion from $O$-prenylated chalcone to $C$ prenylated chalcone via Claisen rearrangement

3-O-Geranyl-chalcone (1) and 3-O-farnesyl chalcone (2) demonstrated potent antifungal activity against Paracoccidioides species, exhibiting MIC values ranging from 0.48 to $3.90 \mu \mathrm{g} \cdot \mathrm{mL}^{-1}$.

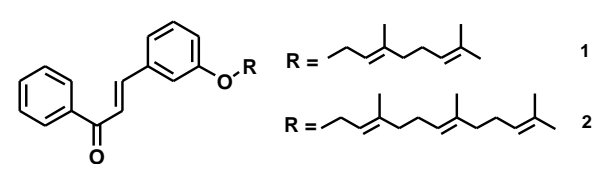

Figure 4. Antifungal 3-O-prenylated chalcones

\section{CONCLUSION}

In conclusion, the current work described the preparation of 20 new chalcones with satisfactory yields. Among these, 3-O-prenylated compounds (1 and 2) showed potent anti-Paracoccidioides activity.

\section{ACKNOWLEDGEMENTS}

CAPES, PROPG, FAPERP, PROAP and Unesp for supporting the present work.

\section{REFERENCES}

${ }^{1}$ Chetana, B. P.; Mahajan, S. K.; and Survana, A. K., J. Sci. Res. 2009, 1, 11.

${ }^{2}$ Dong, X.; Chen, J.; Jiang, C.; Liu, T. and Hu, Y. Arch. Pharm. Chem. Life Sci. 2009, 342, 428. 